\title{
Problem-based learning para la enseñanza universitaria de la Filosofía Medieval
}

\author{
Problem-Based \\ Learning for \\ the Teaching \\ of Medieval \\ Philosophy in \\ University Teaching
}

Problem-

based learning

para o ensino

universitário de

filosofia medieval

\section{Para citar este artículo}

González-García, D. Problem-based learning para la enseñanza universitaria de la Filosofía Medieval. Folios, (54). https://doi.org/10.17227/folios.54-11907

Doctor en Filosofía de la Ciencia, Universidad Nacional Autónoma de México. Profesor de la Universidad del Valle (Cali, Colombia).

Correo: daniel.gonzalez.garcia@correounivalle.edu.co

Artículo recibido $15 \cdot 06 \cdot 2020$

Artículo aprobado $26 \cdot 01 \cdot 2021$ 


\title{
Resumen
}

A partir de una reflexión sobre sus circunstancias en Hispanoamérica, este artículo de investigación sugiere que el conocimiento y la práctica científica regionales acerca del pensamiento medieval podrían beneficiarse de una enseñanza basada en problemas (Problem-based learning). Tras la presentación y análisis de una experiencia didáctica diseñada para estudiantes de pregrado en Filosofía en una universidad pública colombiana, se caracterizan las posibles ventajas formativas, disciplinares, técnicas y profesionales que este enfoque pedagógico puede aportar al estudio de la especialidad, dentro de contextos culturales y geográficos semejantes. Entre ellas, destaca una mayor integración entre un abordaje científico del objeto de los Estudios Medievales y los nuevos requerimientos pedagógicos centrados en competencias.

\section{Palabras clave \\ innovación pedagógica; enseñanza de la filosofía; aprendizaje basado en problemas; educación basada en competencias; educación universitaria en América Latina}

\begin{abstract}
After examining their regional circumstances in Hispanic America, this research paper suggests that a Problem-based Learning ( $\mathrm{PBL}$ ) instruction can improve the knowledge and scientific practices about medieval thought within the region. $\mathrm{A}$ PBL didactical experience designed for BA students at a public Colombian University is presented and analysed as a means of characterizing the disciplinary, technical and professional advantages that could arise from the adoption of such a model in similar cultural and geographical contexts. Remarkable among these gains is a greater integration between a scientific approach to the object of Medieval Studies and the new pedagogical requirements focused on competencies.

Keywords

teaching method innovations; philosophy education; problem-based learning; competency based teaching; higher education in Latin America

Após examinar as suas circunstâncias regionais na América Hispânica, este artigo de pesquisa sugere que uma instrução de Aprendizagem Baseada em Problemas (Problem-based Learning) pode melhorar o conhecimento e as práticas científicas sobre o pensamento medieval na região. Uma experiência didática pbl projetada para estudantes de graduação em uma universidade pública colombiana é apresentada e analisada como um meio de caracterizar as vantagens disciplinares, técnicas e profissionais que poderiam surgir da adoção desse modelo em contextos culturais e geográficos semelhantes. Dentre elas, destaca-se uma maior integração da abordagem científica do objeto dos Estudos Medievais com as novas exigências pedagógicas voltadas para as competências.
\end{abstract}

Abstract

\section{Resumo}

\section{Palavras chave}

inovação pedagógica; ensino de filosofia; aprendizagem baseada em problemas; educação baseada em competências; educação universitária na América Latina 


\section{La Filosofía Medieval en el ejercicio profesional de los filósofos hispanoamericanos}

\section{Antecedentes}

Como ya lo evidenciara D'Amico (2015), aunque en la América hispana los estudios sobre el pensamiento medieval han reseguido el desarrollo de esos mismos trabajos en Europa, están no obstante sujetos a algunas peculiaridades que derivan, entre otras causas, de la lejanía física de los materiales manuscritos y de cierta deficiencia en las técnicas necesarias para analizarlos con éxito. Tras sortear el desdén de los ilustrados y positivistas que pugnaron por adueñarse de las políticas educativas durante el primer siglo de las repúblicas americanas, el resurgimiento de la atención universitaria a las ideas del Medievo estuvo atado en estas latitudes al interés decimonónico por la filosofía tomista, desde una posición militante, mayoritariamente clerical, y encaminada a prolongar o actualizar el pensamiento del Aquinate, a raíz de la encíclica Æterni Patris. Sólo de manera muy lenta y tras sobreponerse a otras agendas teóricas y políticas, las más importantes facultades de la zona vieron nacer grupos de investigación académica para esta especialidad desde mediados del siglo xx. Numerosos esfuerzos traductológicos ampliaron, a su tiempo, el espectro de pensadores y obras examinados por los estudiosos; y el desarrollo de la disciplina parecería estar completo tras la institucionalización de tales preocupaciones en los centros y equipos de trabajo que, por al menos cincuenta años, se han ocupado de transmitirlas a las nuevas generaciones, tanto en instituciones públicas como en las confesionales.

Pero más allá de esa aparente normalidad científica que hoy permite a los medievalistas de la región interactuar en pie de igualdad con los grandes polos europeos replicando los programas y métodos de investigación usuales en el campo, es bien cierto que en Hispanoamérica la Filosofía Medieval tiende a empatarse aún con otra clase de inquietudes; y que suele ser estudiada en función de sus derivaciones o reinterpretaciones, por ejemplo a partir de sus consecuencias en la escolástica de las universidades virreinales de México y Lima, o en diálogo con autores y contextos posteriores y más conocidos por los docentes de la zona. Si bien tales proyectos tienen gran valor intrínseco y permiten comprensiones alternativas del Medievo, absorben a tal punto el interés de los profesores hispanoamericanos que un buen número de ellos sigue abocado a conocerlo y explicarlo desde una instrumentalización que lastimosamente descentra su conocimiento a partir de bases estrictamente técnicas y conceptuales.

La esperanza en que las ideas medievales revistan utilidad hermenéutica para filosofemas remotos (Saranyana, 2004), o su incorporación en programas especulativos encaminados a responder preguntas acuciantes en estos contextos -sobre todo las de corte político y moral- son acaso residuos del cuestionado comienzo de los trabajos filosóficos en la región, y dan cierto tinte apologético a una actividad que no parece tener un acomodo fácil en este ambiente intelectual. A medio camino entre una apropiación vital y la tropicalización de su estudio (D’Amico, 2015), tales visiones sobre el saber de la época reproducen en los estudios medievales ese originalismo que Gutiérrez-Girardot (2002) adscribía al tratamiento hispanoamericano de la Filosofía en general, y los hace avanzar en contravía de un tratamiento exclusivamente técnico de sus objetos y problemas.

\section{Un panorama de la Filosofía Medieval en el currículo formativo}

En los escenarios menos favorables, la investigación y enseñanza del pensamiento medieval de hecho parece una estación forzada en un camino que contempla la Historia de la Filosofía como un esquema organizativo y causalmente conectado donde la relevancia de lo pasado consiste apenas en haber condicionado lo actual. Una revisión sumaria de los programas de estudio en las más importantes universidades de la zona, accesibles en distintos sitios web institucionales, permite constatar que gran parte de los currículos actuales acogen implícitamente la vigencia de tales perspectivas, revelando 
que o bien el conocimiento del Medievo filosófico acusa un profundo rezago fuera de unos cuantos centros, o bien que los logros teóricos alcanzados como resultado de la investigación científica no consiguen una transmisión suficiente en los programas formativos y las lecciones en el aula.

Situada típicamente en los primeros semestres universitarios, la instrucción en Filosofía Medieval suele limitarse en la región a una revisión estandarizada de los autores y tópicos más famosos (Covarrubias, 2012), excepcionalmente desde marcos historiográficos recientes como la visión multicultural favorecida por De Libera (Castello, 2016), pero aceptando todavía en la mayoría de los casos la vieja división entre Patrística y Escolástica, que incluso llega a hacerse sinónimo de la materia misma en la Universidad del Cauca (2013). En todo caso, no resulta insólito que en los planes de estudio y clase de la zona todavía se caracterice al Medievo como una época marcada por la "tensión entre razón y fe, o filosofía y religión” (Redondo, 2018, p. 2); y que se empleen, así sea con valor didáctico, categorías como la de averroismo latino (Pierantoni y Chávez, 2014) que han sido bastante cuestionadas por la historiografía más reciente (Marenbon, 2007).

A esas concepciones inadecuadas se añaden en el aula el frecuente recurso a historias y manuales anticuados (Bréhier, Copleston, Fraile, o el infaltable Gilson); y, en general, un muy escaso contacto con los artículos académicos donde tienen lugar las discusiones más recientes sobre los temas expuestos en los cursos. Común a otros campos del saber universitario y parcialmente imputable a una pobre alfabetización académica de estudiantes y profesores (Carlino, 2003), esa dificultad se aúna a factores más estructurales de la formación universitaria contemporánea que impactan decididamente en la posibilidad de ejercer el estudio de la Filosofía Medieval como un trabajo intelectual meritorio de suyo y sujeto a sus propias reglas disciplinares: preñada de motivos ideológicos, la debilidad en el conocimiento del latín limita, por ejemplo, la absorción de nuevos contenidos, en la medida en que aumenta la dependencia de traducciones a veces centenarias; pero también la viabilidad de emprender indagaciones que aprovechen de lo ya recibido, vista la imposibilidad de los alumnos para ampliar por cuenta propia sus conocimientos y lecturas en fuentes directas de la época. Salvo notorias excepciones, principalmente en Argentina, puede decirse por todo ello que el entrenamiento universitario en Filosofía Medieval no escapa a aquella pobreza técnica que Rossi (1968) consideraba hace medio siglo como el "denominador común de la reflexión filosófica hispanoamericana” (p. Iv).

\section{Retos para la Filosofía Medieval como disciplina universitaria en Hispanoamérica}

Fortalecer la percepción de que el estudio del Medievo es una tarea de la profesión filosófica por derecho propio -y no un mero componente formativo ni un subsidio para interpretar los pensamientos más modernos- demandaría de sus cultores hispanoamericanos la transmisión de contenidos científicos rigurosamente construidos y actualizados, pero sobre todo de los métodos que capaciten a sus aprendices para tomar gradualmente el relevo en esa labor científica. La presencia a largo plazo de esta disciplina en la región como un área relevante de estudio universitario exige por lo menos estas dos condiciones, que sin embargo están lejos de la realidad de las aulas, incluso si en unos pocos centros de investigación el conocimiento del Medievo filosófico goza de cierta solvencia.

La posición curricular de la asignatura, la escasa carga horaria asignada a su estudio en los programas de pregrado o la inmadurez intelectual del auditorio suelen aducirse como las principales limitantes para pretender un entrenamiento más profundo (Priani, 2018), pero se trata de las condiciones inescapables desde las cuales habrá de construirse toda iniciativa que potencie el surgimiento de nuevos medievalistas en nuestro espacio geográfico. Por otra parte, la reciente reconceptualización oficial de la enseñanza universitaria a partir de modelos centrados en las competencias y no en los contenidos, aunada al auge de visiones pedagógicas que privilegian la experiencia escolar del estudiante por sobre el conocimiento 
de los objetos, entraña desafíos adicionales al modo usual de transmitir la Filosofía Medieval y generar interés en su exploración por parte de las nuevas generaciones.

Bien afrontadas, estas condiciones suponen sin embargo una oportunidad para superar las deficiencias técnicas del medievalismo hispanoamericano, y podrían favorecer la profesionalización de futuros profesores e investigadores de esta disciplina. Si ante esas necesidades ineludibles la formación universitaria en el área aprovecha las nuevas posibilidades didácticas como lo hace ya con otras innovaciones tecnológicas, acaso consiga superar el aspecto anquilosado y repetitivo de los currículos o la estandarización de sus contenidos en el salón de clases, principales estorbos para expandir o consolidar las preocupaciones teóricas de los noveles filósofos y alentar su vocación como medievalistas.

Y es que más que como un quehacer científico ocupado de plantear y resolver cuestiones intelectuales aún inexploradas, la enseñanza de la Filosofía Medieval en la región lleva a pensar y valorar esta disciplina como un saber minoritario, derivativo y no siempre interesante, cuyas agendas de investigación están diseñadas siempre en otra parte y para propósitos teóricos e históricos que por principio son ajenos a quienes están obligados a estudiarla. El remedio a este hecho no parece provenir, sin embargo, de un chovinismo adaptativo empeñado en entender lo medieval desde la circunstancia y los horizontes americanos: medir la relevancia o el interés de un asunto científico en función de su inmediata relación con el contexto de los investigadores o sus públicos es tan perjudicial para una disciplina académica como olvidarse de que los conocimientos transmitidos han de posibilitar también el ejercicio eficiente y conceptualmente riguroso de la profesión con independencia del contexto geográfico, en el marco de una comunidad científica internacional que se articula en torno a los métodos y a los temas investigados.

Las experiencias y estrategias formativas de saberes que en el pasado enfrentaron disyuntivas semejantes, pero superaron la brecha entre un estado avanzado del conocimiento y una transmisión efectiva de éste en las aulas universitarias, pueden resultar ilustrativas sobre cómo evitar esos escollos. Aun a título experimental, dichos precedentes necesitarían ser evaluados como medios para integrar mejor los resultados científicos conocidos, las condiciones reales de la práctica investigativa, y la preparación de los futuros medievalistas hispanoamericanos a partir de una transmisión de contenidos más efectiva, pero sobre todo por la adquisición en el aula de las técnicas pertinentes al ejercicio de esa profesión como un quehacer académico. Con ese objetivo a la vista, el presente artículo examina la plausibilidad del modelo de aprendizaje basado en problemas (Problem-based learning) como una alternativa didáctica para la formación universitaria en Filosofía Medieval en el ámbito hispanoamericano. A partir de los resultados de esa experiencia de enseñanza, se reflexiona sobre cómo ese modelo perfeccionaría y tecnificaría la formación de este campo del quehacer académico en la región.

\section{Caracterización general del modelo didáctico PBL}

El Problem-based learning ( $\mathrm{PBL}$ ), o aprendizaje basado en problemas (ABP), es un enfoque pedagógico derivado de los trabajos que Barrows, Tamblyn y otros emprendieran a mediados de la década de los sesenta para mejorar la educación médica, que por entonces atravesaba dificultades afines a las ya descritas, principalmente un distanciamiento entre la formación en el aula y las condiciones cotidianas de la práctica clínica (Gallagher, 1997). Muy socorrido desde entonces en la enseñanza de la Medicina, se ha aplicado con éxito en la educación básica o K-12 en países asiáticos con alto logro escolar (Peen y Arshad, 2014). Su introducción en Hispanoamérica suele acompañar al International Baccalaureate, que privilegia en su currículo las aproximaciones constructivistas y basadas en la investigación (Li, 2012). Todavía se emplea muy poco en las universidades de Hispanoamérica, y prácticamente nunca para la enseñanza de la Filosofía en el nivel universitario. 
El enfoque PBL se fundamenta en el planteamiento reiterado de interrogantes (inquiries) suscitadas por un objeto detonador (trigger) que a menudo toma la forma de un caso o situación para el estudio. Dicho precursor presenta al estudiante apenas una dificultad difusa o débilmente estructurada (ill-structured) que espera desatar su curiosidad sin restringir de antemano el tema o el resultado del trabajo (Gallagher, 2009). Durante el proceso, los alumnos adquieren tanto las habilidades como los conceptos disciplinares que les permiten relacionar de modo relevante las inquietudes investigativas entre sí y con la evidencia, quedando en capacidad de integrar a ese núcleo adquirido la nueva información a medida que ésta se necesita o aparece, incluso después de completado el tiempo asignado al aprendizaje de la materia en el currículo escolar.

Semejante flexibilidad instruccional -favorable al desarrollo de la creatividad, el autodidactismo y el pensamiento crítico- demanda por contraparte un instructor o equipo docente con amplia disponibilidad para guiar y acompañar a los aprendices; un cambio en la valoración del oficio del profesor (que pasa de proveedor a árbitro o garante de la información); y, en general, una mayor apertura hacia la aparición de conocimientos que podrían exceder la planeación estricta de lecciones según los modelos habituales. La pericia del instructor en el campo de intereses explorado por los estudiantes es asimismo un factor indispensable de este modelo, pues constituye el límite más robusto frente a la arbitrariedad de las ideas construidas por el estudiante, y permite lidiar de modo seguro con la gran variabilidad del proceso didáctico (Gallagher, 2009; Williams, 2012; Leary, Walker, Shelton y Fitt, 2015).

Aunque una interpretación negligente de PBL podría deducir que el maestro resulta fútil ante la creciente autonomía educativa posibilitada por el modelo y la amplísima disponibilidad de recursos informativos que potencialmente exceden el saber de cualquier docente concreto, este último tiene en realidad un papel más activo que en el aula tradicional, y si bien queda descentrado como fuente del saber adquirido por los alumnos (McCaughan,
2015), siguen siendo exclusivamente suyos el dominio de los elementos estructurales del curso, la definición de los objetivos, el diseño de las unidades didácticas, la anticipación de las rutas seguidas por los discentes, y la continua vigilancia y diálogo con estos para enfocar sus preocupaciones o acrecentar sus competencias metacognitivas o heurísticas (Goh, 2012).

Como puede inferirse de lo dicho, el funcionamiento óptimo de PBL ocurre entonces en tutorías para pequeños grupos culturalmente homogéneos, cognoscitivamente maduros, ampliamente informados y susceptibles de un estrecho seguimiento didáctico. ¿Conviene, entonces, a las circunstancias de enseñanza de la Filosofía Medieval detalladas arriba? ¿Es viable en un contexto donde el grueso de la educación superior en Filosofía depende de universidades públicas en condiciones casi opuestas a estas ideales? ¿Qué beneficios potenciales aportaría una instrucción PBL a la formación de futuros investigadores de la Edad Media? Para contribuir al análisis de tales cuestiones, se detallan enseguida el modo, la dinámica y los resultados de un curso que ha ensayado la implementación de ese enfoque para la enseñanza de la Filosofía Medieval en un ambiente universitario típico de Hispanoamérica.

\section{Un curso experimental PBL de introducción a la Filosofía Medieval}

\section{Contexto instruccional y demográfico de los participantes}

Situada en el segundo semestre de los programas de Licenciatura y Profesional en Filosofía de la Universidad del Valle en Cali, Colombia, la Introducción a la Filosofía Medieval es un curso obligatorio en la formación de profesionales y licenciados en Filosofía. Tiene una carga horaria de tres horas semanales durante un período que normalmente se extiende por 16 semanas. En vistas de un modelo de flexibilidad curricular adoptado por el Departamento de Filosofía en esa institución, su seguimiento no supone continuidad alguna con cursos previos ni posteriores; es decir, no existe un 
conocimiento previo que el estudiante deba acreditar antes de incorporarse al curso de esta materia.

Durante el último lustro, la asignatura ha recibido un promedio de 80 estudiantes por año. Las estadísticas demográficas ofrecidas por la propia Universidad del Valle (2017) para su población general son también representativas del perfil de los alumnos de esa clase: estos provienen mayoritariamente del suroccidente colombiano, sobre todo de los departamentos de Valle del Cauca, Cauca, Nariño y Huila. Un tercio de ellos se identifica como indígena o afrodescendiente. Hasta un $90 \%$ reside en viviendas de los tres estratos menos favorecidos de la pirámide socioeconómica oficial. Unos siete de cada diez tienen acceso domiciliario a un computador con internet, y provienen de hogares donde sólo un $14 \%$ de sus padres cuenta con estudios universitarios.

En los años 2017 a 2019, la usualmente numerosa cohorte se dividió en dos grupos de aproximadamente 40 estudiantes, cada uno a cargo de un profesor definitivo con experiencia mínima de una década en la docencia universitaria. Durante el segundo semestre de 2018 (agosto de ese año a mayo del año siguiente, un período anómalo en duración por circunstancias de orden público) una de tales secciones fue conducida usando el enfoque didáctico PBL. Originalmente conformada por 36 estudiantes, 19 de los cuales eran varones y 17 mujeres, esa porción de la cohorte quedó dividida a su vez en ocho equipos de hasta cinco integrantes. Tras una interrupción extraoficial de las actividades académicas -frecuente, por lo demás, en esa universidad-, los equipos sufrieron algunas reorganizaciones para acomodar a los 13 alumnos que culminaron el semestre.

\section{Dinámica del curso}

$\mathrm{Al}$ inicio del período escolar, cada equipo recibió como detonador una carpeta con la imagen de por lo menos tres folios de manuscritos medievales ilustrados, procedentes de colecciones digitales, y mayoritariamente del acervo de la British Library. Las imágenes fueron intencionalmente seleccionadas por el profesor en función de su pertinencia para los principales objetivos didácticos (posibilitar la construcción rigurosa de conocimiento medieval a partir de sus fuentes y confrontar las convicciones previas y presentes sobre la época), y agrupadas por su similitud temática o sus posibles connotaciones filosóficas. Los materiales fueron acompañados de un descriptor de aproximadamente 75 palabras. Sin aportar información específica sobre lo representado en las láminas, este párrafo aludía con enunciados generalísimos a las visiones vulgares del Medievo como una época ignorante o culturalmente atrasada, en franco contraste con lo que las llamativas imágenes mostraban (para una lista de estas imágenes y sus respectivas presentaciones, véase anexo). En la redacción de los descriptores se tuvo cuidado de no incluir ningún lenguaje técnico, a fin de que fuesen comprensibles para un egresado promedio del bachillerato.

Las primeras dos jornadas del curso se dedicaron a presentar a los estudiantes la metodología PBL, y a orientarlos sobre el proceso y los resultados esperados usando como ejemplo un artículo especializado donde se discute la revaloración de una miniatura del manuscrito Londres, Royal 6 E vi, frecuentemente empleada para ilustrar publicaciones sobre la peste negra (Green et ál., 2014). Tras esto, las siguientes sesiones fueron conducidas a través de un ciclo reiterativo de siete pasos centrado en que los estudiantes extrajeran información de los folios asignados. Esos estadios pueden describirse como a) discusión exploratoria de datos, a modo de una tormenta de ideas; b) enumeración de las certezas o datos firmes ya conocidos por el equipo; c) formulación de preguntas acerca de dichos datos y reconocimiento de las nuevas necesidades de saber, formuladas a manera de preguntas (¿qué?, ¿por qué?, ¿cuándo?, ¿cómo?); d) discusión analítica de los elementos de tales interrogantes, de modo grupal y con la interacción del profesor como guía y árbitro de la precisión terminológica o la viabilidad científica de las cuestiones planteadas; e) registro de posibles soluciones a las interrogantes planteadas; f) discusión programática que estableciera la agenda para el paso posterior; g) investigación para el acopio de nueva información. 
Este último paso se cumplía principalmente fuera del aula, a partir de un uso cada vez más selectivo y especializado de recursos informáticos. Se partía, por ejemplo, de una noción general basada en diccionarios o wikis, para avanzar hacia un uso progresivo de bases de datos académicas. Los materiales acopiados tras esas búsquedas debían ser procesados por cada equipo antes de la siguiente sesión y servían como punto de partida para reiniciar el ciclo en la siguiente semana. Toda la actividad del semestre era obligatoriamente asentada, según los pasos mencionados, en una bitácora que puntuaba para la calificación final.

Los primeros contactos con los folios causaban en los estudiantes una sensación de extrañeza, pues una muy débil formación previa en Historia Universal, típica de la educación colombiana hasta antes de la Ley 1874 del 27 de diciembre de 2017, les impedía reconocer o describir con suficiente riqueza los elementos representados, $\mathrm{o}$ asociarlos siquiera a un preconcepto vago de la Edad Media. Desde ese pasmo inicial, a veces frustrante, algunos estudiantes consiguieron identificar correctamente algunos elementos gráficos aislados usando aplicaciones para el reconocimiento visual, mientras que otros -con mayores habilidades digitales- arribaron incluso a la ficha catalográfica de cada manuscrito en el sitio web de la biblioteca de proveniencia. Mecanismos de traducción automática permitieron que el escaso dominio de otras lenguas, predominante en alumnos del mencionado perfil demográfico, no fuese obstáculo para apropiarse de los datos encontrados.

La información obtenida por esta vía y por otras más tradicionales -como la consulta de enciclopedias, libros sobre la época medieval y otras fuentes impresas- debía en cualquier caso ser categorizada o agrupada en función de sus semejanzas. En este punto los alumnos eran continuamente exhortados a abandonar la pura descripción de elementos individuales ("una figura de un ser superior con rasgos de león sentado en la estructura de una iglesia" refiriéndose al Pantocrátor que preside el esquema de la Sphaera de Pecham en el manuscrito Arundel $83 \mathrm{f} .123 \mathrm{v}^{\circ}$ ), para dar lugar a enunciados gradualmente más abstractos, integradores y complejos, formulados con términos específicos de la disciplina y la época. A medida que esa exactitud léxica aumentaba, era posible que los componentes de tales oraciones se incorporaran a preguntas susceptibles de ser investigadas bajo una perspectiva estrictamente técnica. Idealmente, después de este refinamiento los alumnos debían ser capaces de rastrear los elementos de solución a sus interrogantes en la literatura especializada que localizaban tras búsquedas gradualmente más precisas y ricas en bases de datos y repositorios institucionales.

Para conseguir paulatinamente este fin, desde la tercera sesión del curso el profesor se incorporaba por momentos al trabajo de los equipos, cuestionando los avances presentados, la formulación de las preguntas o la pertinencia de sus elementos y términos. Ocasionalmente, el docente sugería de modo indirecto algunas vías para reconducir la investigación ulterior, pero en una primera fase sólo por excepción mencionaba explícitamente conceptos de la época o vocabulario técnico, con miras a no condicionar la búsqueda de datos ni la definición del plan investigativo. Se procuraba, en cambio, que los estudiantes se formaran un criterio para el descubrimiento y criba de datos que les permitiese adquirir, por cuenta propia y en la medida estrictamente necesaria, el léxico necesario para sustentar sus ideas. En todo caso, se prestaba especial atención a que estas últimas se expresaran siempre con los vocablos más precisos posibles, forzando a su revisión y redefinición continuas. De esta suerte, una primera formulación tentativa del problema como "la importancia del animal en la Edad Media" se enriqueció y precisó para desembocar en una pregunta sobre "la moralización de los hábitos animales a partir del Physiologus y su relación con la literatura ejemplarizante".

El mismo proceso era repetido hasta que, a juicio del docente experto, los alumnos hubiesen alcanzado un problema de investigación; esto es una interrogante suficientemente abstracta y definida para su nivel cognoscitivo que fuese apta para entrar en diálogo con los textos y los resultados 
comunicados por la literatura científica que ellos debían discriminar en función de la utilidad para responder a su propia pregunta. Tras esta validación -alcanzada hacia el tercer mes de trabajo- se reforzó la exigencia de que las informaciones provistas como base para la discusión cotidiana en el aula ya sólo proviniesen de por lo menos un texto propiamente medieval, y de artículos académicos o libros técnicos. Las estrategias mínimas para la adecuada localización, registro y manejo de estos materiales fueron transmitidas durante una sesión grupal, pero la selección y lectura dependieron enteramente de los equipos de estudiantes, quienes tuvieron que lidiar por su cuenta con el hecho de que el grueso de los textos relevantes no estaba redactado en castellano, y que muchas de las obras de la época mencionadas en los artículos carecían de versiones a algún idioma moderno que fuese susceptible de la traducción robotizada empleada antes con las páginas de internet y los materiales impresos.

Esta segunda fase del curso resultó la más demandante para todos los participantes en cuanto a la necesidad de reuniones personalizadas fuera del aula, en los espacios y durante tiempos asignados por la Universidad para la atención a estudiantes (hasta cuatro horas semanales). Fue también el motivo para la deserción de ocho de los veintiún alumnos todavía inscritos tras las cancelaciones administrativas de la matrícula. Algunos de los grupos originales se desintegraron por completo en este punto, de manera que cuatro de los trece supervivientes debieron trabajar en solitario. Dichas asesorías se enfocaron a discutir los aspectos más técnicos de las investigaciones, lo cual ofreció la oportunidad de minilecciones (Gallagher, 2009; Bender, 2012) que satisfacían curiosidades lingüísticas y paleográficas; o dudas conceptuales bastante más sofisticadas, resultantes de la vinculación entre los datos conocidos y el problema en examen. Las cuestiones de investigación se reformularon en consecuencia, hasta alcanzar una cota que hacía esperable fundamentar una respuesta a ellas en el tiempo restante del curso por medio de los materiales textuales trabajados hasta entonces. Las formulaciones concretas de los estudiantes en esta versión del curso se detallan en la sección "Formulación, resolución y valoración de los problemas de investigación”.

En la tercera y última fase, de unas tres semanas, los equipos se implicaron en el perfeccionamiento de sus respuestas, y en el diseño de exposiciones audiovisuales de veinte minutos que de manera oral a) diesen cuenta del proceso para descubrir y refinar del problema, b) presentaran este último mediante una formulación clara y terminológicamente correcta, c) ensayaran una respuesta con fundamento en los textos de la época y en la literatura secundaria leídos por ellos y d) expresaran algunas dificultades derivadas que merecieran un mayor análisis o pudiesen ampliar los horizontes de la investigación. Esta presentación final estaba pensada, además, como la revisión final o debriefing del proceso mismo de aprendizaje, y como oportunidad para complementar la información o proponer vínculos entre todas las investigaciones presentadas por el grupo, según recomiendan los teóricos del enfoque PBL (Peterson, 1997).

\section{Evaluación y rúbricas}

Al inicio del semestre se planteó, para todas estas actividades, un esquema de evaluación que mediante un formulario automatizado recabara la percepción que del desempeño de cada alumno expresaban él mismo, sus compañeros de equipo y el profesor (evaluación $360^{\circ}$ ). Esta nota representó aproximadamente la mitad de la calificación final y valoraba la orientación a resultados, el compromiso con el aprendizaje y la cooperación con el trabajo colectivo. Constituía, además, un registro testimonial del avance y necesidades desde la óptica de los propios estudiantes. El resto de la calificación del semestre dependió del proceso de la investigación misma (registrado en la bitácora) y de los resultados expuestos. Se puntuaba la calidad y pertinencia de estos (atendiendo a su originalidad, coherencia demostrativa, especificidad filosófica y posibilidades de expansión); su fundamentación (soporte bibliográfico, soporte conceptual, verificabilidad); y los aspectos retóricos de su defensa (materiales de apoyo, expresión, respuestas, efectividad ante el 
auditorio), con la pretensión de familiarizar a los estudiantes con algunos de los tipos discursivos de la comunicación científica, como la ponencia o el póster. Para determinar estos rubros, el profesor tuvo en mente no la certificación de que los estudiantes hubiesen meramente asimilado datos sobre el Medievo y sus ideas, sino que los usaran para plantear una pregunta filosófica significativa dentro de dicho contexto histórico y en el marco de la disciplina. La pregunta debía permitir, además, la explicación de algunos aspectos de las imágenes recibidas al principio del curso ante un auditorio de pares.

\section{Formulación, resolución y valoración de los problemas de investigación}

Como resultado del proceso descrito, los equipos y estudiantes individuales que concluyeron el curso llegaron a plantear por cuenta propia, con diversa profundidad y éxito, siete problemas de investigación cifrados en las siguientes preguntas:

1. ¿Cómo influyeron la tradición médico-filosófica de la época y el análisis experimental en la divulgación de las causas de la pestilencia realizada por Jacme de Agramont?

2. ¿Cómo mediaron las instituciones y las traducciones de la Edad Media en la conservación del saber [astronómico] antiguo?

3. ¿Por qué el hombre medieval, interesado en la existencia de Dios, investiga la totalidad de lo creado (universum)?

4. ¿Cómo era la relación de otredad de los europeos medievales con los extranjeros?

5. ¿Cómo adaptaron Boecio y Hugo de San Víctor el sistema de educación clásica a la formación cristiana?

6. ¿Cuáles son, desde la teoría agustiniana, las relaciones entre signo y significar en el cómputo manual, la mano guidoniana y la meditatio per manus?

7. ¿Cómo, a pesar de la teoría de las seis edades, la Edad Media pudo mantener una concepción unitaria de la Historia?
Para el trabajo de análisis y respuesta, los alumnos acudieron a algunos textos medievales frecuentemente empleados en cursos similares (el De Trinitate, el De magistro o el De doctrina christiana de san Agustín; las Etymologiae de san Isidoro; o el Didascalicon de Hugo de San Víctor); pero también a obras menos estudiadas, como el Regiment de preservació de pestilencia, de Jacme de Agramont; el Prologus in Eptateuchon, de Teodorico de Chartres, o el Sermo inauguralis, de San Buenaventura, a las cuales los estudiantes arribaron desde las referencias de la bibliografía erudita empleada para discutir y analizar sus interrogantes. El ejercicio supuso, pues, una de sus primeras oportunidades para experimentar el tipo de tarea que debería fundamentar su formación universitaria, pues los llevó a encauzar una curiosidad o perplejidad inicial hacia una verdadera cuestión de investigación para la cual pudieron plantear soluciones en el marco de la especialidad en la que se forman.

Aunque la duración del curso y su novedad metodológica, aunadas a la edad y los conocimientos previos de los participantes no permiten sorpresas en cuanto a la calidad de las soluciones alcanzadas, éstas sí representaron de manera incipiente el tipo de actividad derivada del proceso profesionalizado de investigación que todo estudiante de Humanidades debe sostener por lo menos hasta el trabajo de tesis. Al final del trabajo del semestre, los propios estudiantes llegaron a reconocer que, no obstante las exigencias y dificultades inherentes a ese modelo de aprendizaje, se encontraban más satisfechos con los resultados de su propio empeño; y que valoraban más los conocimientos así obtenidos que los aprendidos en otros cursos del mismo período lectivo.

\section{Discusión}

\section{Ventajas percibidas de la aproximación PBL en la enseñanza de la Filosofía Medieval}

A diferencia de la evaluación tradicional por medio de pruebas parciales, controles de lectura o reportes finales, la construcción paulatina de investigaciones según el modelo PBL permitió a los estudiantes de 
ese curso integrar de manera sensata los datos que ellos mismos se procuraban, hasta constituir un problema rico del cual se apropiaron como un reto intelectual. Sobre todo entre los jóvenes, ese enfoque didáctico parece aportar cierta dimensión lúdica a la adquisición de conocimiento. La percepción de que el instructor confía en su capacidad para despejar esa dosis de misterio contribuye, además, a mejorar el aplomo con el que enfrentan la ambigüedad y la complejidad propias de toda tarea científica. Durante las asesorías personalizadas, los estudiantes llegaron a manifestar espontáneamente este hecho ya reportado por la literatura (Gallagher, 1997).

La imposibilidad e inutilidad de buscar soluciones prediseñadas a la clase de preguntas teóricas construidas con este tipo de ejercicio supuso también un cambio en su percepción del logro escolar: contra el hábito de rastrear en los manuales o apuntes de clase unos fragmentos o ideas para presentarlos como la respuesta correcta durante exámenes que evalúan la memorización o absorción de doctrinas concretas, la instrucción basada en problemas obligó a los estudiantes a evaluar críticamente esas porciones textuales, y subordinarlas a la demostración de una hipótesis planteada por ellos mismos. Esto parece haber incrementado su capacidad interpretativa (en proporción, desde luego, a su madurez psíquica o su entrenamiento previo), pero además entraña una buena simulación del oficio que piensan desempeñar en el futuro, como docentes e investigadores en el campo de la Filosofía.

Asimismo, el contacto con una Edad Media mucho menos mediada por conferencias, temarios e historias panorámicas parece haber contribuido a sensibilizar a los estudiantes sobre la complejidad y requerimientos técnicos de un estudio fundamental de la época, y la importancia primordial que en él tiene el contacto directo con las fuentes. La presencia permanente de los folios manuscritos como referente básico para la construcción de los problemas de investigación supuso, por ejemplo, la oportunidad de mostrarles de primera mano la razón por la cual no puede conocerse a fondo la Filosofía del Medievo sin dominar el latín o la paleografía; y hasta qué punto la comprensión del pensamiento de la época necesita recurrir a otro tipo de saberes y datos, como las convenciones iconográficas, el funcionamiento de dispositivos tecnológicos o las prácticas litúrgicas de la época, confirmando así la afirmación de Zumthor (1980) en el sentido de que todo documento medieval reposa sobre el complejo tejido de su tiempo; y que debe ser re-emplazado sobre este para obtener de él una comprensión adecuada.

El planteamiento mismo de las preguntas y los incipientes intentos de solución obtenidos en el curso atestiguan, en suma, la posibilidad de conseguir, sin necesidad de demasiados cambios en la estructura institucional, sino apenas en el diseño instruccional, unos alumnos mejor entrenados para convertirse en agentes de la construcción de conocimiento, y no en simples receptores o repetidores. El enfoque PBL parece facilitar, en este caso, una adquisición directa y circunstanciada de la terminología técnica, pues la necesidad de aprender tal o cual vocabulario para describir las realidades conceptuales del Medievo no se determina de antemano ni en abstracto, sino en el horizonte de un problema preciso, y siempre con un obvio sentido funcional que favorece su retención a largo plazo.

\section{Algunas dificultades para la implementación del modelo}

A pesar de las ventajas mencionadas, los participantes de este curso experimental reportaron también algunas limitaciones sobre las cuales debe llamarse la atención del resto de la comunidad docente y científica si el modelo quisiera implementarse como una opción didáctica a futuro. Por un lado, en las primeras fases del proceso, resultó especialmente difícil para algunos estudiantes superar el anacronismo en la selección del lenguaje con el que describían las ilustraciones: se empeñaban, por ejemplo, en hablar de órbitas elípticas y telescopios ante el esquema de Pecham, o insistían en referirse a los signos zodiacales como superstición. El empeño por explicar lo que observan sólo en términos de sus propios hábitos y juegos terminológicos, o con base 
en el poco léxico filosófico adquirido previamente y que deriva sobre todo de pensadores contemporáneos, puede constituir uno de los mayores obstáculos al éxito del proceso aquí presentado. En estudiantes menos maduros, es de hecho una fuente continua de frustración que los mueve a abandonar el intento. Las causas profundas de este hecho parecen ser estructurales, y quizá dependan de una visión politizada y positivista de la Historia, muy frecuente en la educación hispanoamericana. Aunque un cambio definitivo al respecto no es esperable en tanto no se fortalezca una mejor enseñanza de esta última disciplina en la educación básica de la zona, resulta posible enfrentar la dificultad en el plano inmediato y para los fines de cursos como éste si se desnuda ante el estudiante el carácter relativo de toda presentación historiográfica, y si se le invita a cuestionar la razones y fundamentos del saber que ha recibido acerca del pasado. En vistas del momento vital de la mayor parte de los participantes de asignaturas como ésta, la tarea puede abordarse con relativa facilidad.

Por otro lado, la falta de traducción a lenguas modernas para gran parte de los materiales medievales referidos como relevantes en la literatura secundaria fue explícitamente identificada por los estudiantes como otro obstáculo que les impidió obtener resultados más ricos. Ellos mismos se encontraron perplejos ante las pocas salidas a este problema, y experimentaron directamente la notoria pérdida del conocimiento de la lengua latina, aun entre quienes por su profesión deberían poseerlo. Como la anterior, esta es también una dificultad profunda, y deriva de una negligencia con la formación clásica, tanto en la educación básica como en los departamentos de Humanidades. Contra esa desventaja, el remedio a corto plazo parece consistir en fomentar la proliferación de traducciones castellanas de textos medievales por quienes aún están en capacidad de realizarlas. Esa tarea, otrora muy valorada, ha decaído en la medida en que tiende a considerarse la traducción como un trabajo derivativo, e inferior incluso a la escritura de artículos sobre temas manidos. Como un auxilio a la formación de sus futuros colegas, los medievalistas ya consolidados podrían procurar, entonces, el aumento en la cantidad de textos que por su dimensión, complejidad y tema puedan ser fácilmente abordados por quienes se introducen al estudio de esta disciplina. En cursos como este, contar con un amplio abanico de opciones fácilmente localizables resulta de mucha ayuda como parte del entrenamiento filosófico en tanto las condiciones de enseñanza del latín no sean mejores.

Finalmente, la dificultad de comprender e interpretar alguna literatura académica excesivamente erudita supuso para los participantes de este curso un reto adicional, que, sin embargo, no debería atenuárseles en futuras versiones: por cuanto patentiza los requisitos para acceder a la discusión especializada de un asunto, este obstáculo ofrece a los alumnos una impresión realista de los retos propios de la actividad científica y -a diferencia de una percepción simplificada de la investigación en Historia de la Filosofía como un tedioso acopio de datos y doctrinas provenientes de una obra panorámica- contribuye a prestigiar los estudios medievales como una labor exigente y fértil. De hecho, la impermeabilidad inicial que todo artículo científico presenta para los no especialistas ha sido sugerida como un detonador del aprendizaje disciplinar cuando se toma ella misma como problema, en el sentido que este término tiene para el enfoque PBL (White, 2001), y podría ser ocasión adicional de aprendizaje, no sólo de temas particulares sino del propio proceso de lectura académica (literacy). Debido a ello, y de manera análoga a lo que ocurre con la traducción de obras medievales, garantizar el acceso fácil a los resultados de investigaciones suficientemente diversas, que cubran un mayor número de autores, textos y problemas, cumple también una utilidad formativa para los estados iniciales de la profesión filosófica.

Esta última posibilidad es pocas veces tenida en cuenta por los investigadores, quienes suelen pensarlos principalmente como medios para comunicar a pares los resultados de sus pesquisas, pero la función de esos materiales en cursos como el aquí presentado constituye prueba de otras utilidades potenciales que deberían ser ponderadas al momento de decidir 
para quién o cómo escribirlos, y por qué medios distribuirlos. El esfuerzo más inmediato en este rubro consistiría en facilitar la difusión digital y el acceso abierto a esos trabajos, pues si bien resulta sencillo hacerse clandestinamente incluso con publicaciones muy costosas, la difusión en repositorios gratuitos o en las redes profesionales de los propios autores es una alternativa legal poco explotada que (sobre todo para trabajos de escasa corrida editorial) complementaría muy bien el esfuerzo de cursos PBL sobre el tema, e incrementaría el prestigio y la vigencia de los propios investigadores de la Filosofía Medieval.

\section{Posibles aportes de PBL para el estudio de la Filosofía Medieval en Hispanoamérica}

Si bien resulta temerario pretender conclusiones definitivas desde una sola experiencia, los resultados de este primer ensayo de una Introducción PBL a la Filosofía Medieval animan a pensar en nuevos derroteros para asignaturas semejantes, incluso en los contextos académicos que han sido refractarios a tales estudios. La facilidad con la cual este modelo integra la exploración propiamente científica de un objeto con los nuevos requerimientos pedagógicos, y el modo como ayuda a entrenar a los estudiantes en habilidades profesionales específicas -según lo demandan las reformas educativas recientemente emprendidas en varios países- permite mantener cierto optimismo respecto a la pertinencia de la asignatura ante la presión que sobre ella suelen ejercer esta clase de cambios curriculares modernizantes. El modelo de enseñanza PBL parece ser útil para mejorar las condiciones de estudio de la Filosofía Medieval en la América hispana en aspectos de orden instruccional, disciplinar y formativo, y podría tener asimismo un efecto en la profesionalización de estos quehaceres en la región.

En la medida en que enfrenta de manera temprana a los estudiantes con las condiciones reales y cotidianas del trabajo especializado, involucrándolos sin ficciones en los procesos de construcción de conocimiento, la aproximación PBL fortalece la instrucción desde una especificidad técnica que es difícil alcanzar por medio de pedagogías magistra- les. Y es que, si bien las lecciones de ese tipo pueden resultar enormemente ilustrativas de los logros en una determinada rama del quehacer filosófico, suelen encubrir las tareas implícitas en la generación de los conocimientos que se transmiten.

Asimismo, como los contenidos de un curso PBL son determinados primariamente por los intereses y respuestas manifestados por los estudiantes ante la evidencia que se les ofrece, la instrucción de la Filosofía Medieval según este modelo alienta una ampliación y diversificación del canon vigente en estas asignaturas universitarias; y obliga a los docentes a expandir sus propias áreas de trabajo. Este hecho tiene un alcance disciplinar pues parece promover actualización continua del cuerpo docente, y parece comportar también una ocasión para aumentar la visibilidad e impacto de los logros científicos obtenidos por los medievalistas hispanoamericanos, inicialmente dentro de la propia región. Para las facultades y centros con tradiciones más sólidas de investigación sobre el tema, un aumento en la demanda de materiales científicos que puedan ser empleados para una enseñanza basada en problemas es una oportunidad inmejorable de aumentar su influencia intelectual.

Desde el punto de vista formativo, como el enfoque PBL favorece el contacto de los aprendices con las evidencias crudas requeridas para estructurar verdaderos problemas científicos, los docentes que lo empleen pueden adquirir una mayor conciencia sobre las necesidades de preparación técnica de los estudiantes a su cargo; y tomar a ese respecto las decisiones necesarias ya en el marco inmediato de su propio curso, ya promoviendo modificaciones curriculares de mayor calado. Hace menos de veinte años el examen de los datos básicos para este tipo de estudios solía implicar todavía la adquisición de costosas ediciones importadas o viajes de estudios por bibliotecas europeas. Hoy los manuscritos y otros recursos son fácilmente accesibles, pero esto no los hace sin embargo abordables, a pesar de la mucha curiosidad que causan entre los estudiantes. Para que esta difusión sin precedente de materiales medievales conlleve un aprovechamiento adecuado 
por parte de las academias americanas, resultaría ineludible fortalecer la formación de docentes y estudiantes en paleografía, codicología, idiomas clásicos y manejo informático de los corpora de textos. Tales necesidades, reveladas y parcialmente solventadas por iniciativas didácticas como ésta, son acaso un punto crítico que debe atenderse cuanto antes para que la formación regional en Filosofía Medieval esté a la altura de la circunstancia presente.

Por último, en cuanto hace a la calidad profesional de los medievalistas de la región, el enfoque PBL parece tener utilidad como una herramienta para detectar tempranamente los mejores talentos en esta clase de estudios, e incorporarlos a las iniciativas académicas y las oportunidades laborales. Empleado a menudo como un recurso pedagógico para los estudiantes más dotados, la perspectiva PBL facilita a los profesores la identificación clara de los estudiantes más capaces de hacer preguntas profundas y construir conocimientos relevantes, lo cual permite discriminar a los más autónomos y mejor provistos de las habilidades metacognitivas que indican talento para la carrera científica (Barrows, 1988; Gallagher, 1997). El seguimiento cercano y la atención formativa a estos alumnos promisorios redundaría en una academia más numerosa y fuerte, capaz de arraigar en Hispanoamérica el estudio de la Filosofía Medieval por sí misma y de acuerdo con los estándares técnicos más altos.

\section{Anexo. Folios manuscritos y descriptores empleados para instrucción PBL en el curso Introducción a la Filosofía Medieval 2018-2 en la Universidad del Valle en Cali}

Carpeta 1. Proveniencia: Londres, Bibl. brit., mss. Harley 5311, ff. $2 \mathrm{v}^{\mathrm{o}}, 5 \mathrm{v}^{\mathrm{o}}, 10 \mathrm{v}^{\mathrm{o}}$; Add. 17358, ff. $2 \mathrm{v}^{\mathrm{o}}$. $8 r^{\circ}$. Descriptor: "Si bien suele pensarse que la Edad Media hizo del conocimiento el patrimonio de unos cuantos clérigos recluidos en monasterios y abadías, hay abundantes testimonios de la difusión y empleo cotidiano del saber científico, como también ocurre en nuestra época. La confección y comercio de pequeños libros con información puntual sobre temas de utilidad inmediata ayudó a sacerdotes, médicos o campesinos para desempeñar su labor de una manera exitosa e informada".

Carpeta 2. Proveniencia: Londres, Bibl. brit., mss. Add. 24189 f. 9vo; Royal 6 E IX, ff. 22 ro 24 vo. Descriptor: "Habituados a concebir el transcurso de la Historia como una puesta en escena de tres actos, solemos creer que la Edad Media rompió radicalmente con la Antigüedad, sus costumbres, logros o ideas. Por el contrario, el hombre medieval se consideraba a sí mismo como un eslabón más en una larga cadena de empeños que le permitía perfeccionar las aportaciones de sus predecesores, los 'gigantes' muchas veces anónimos a cuyos hombros nos elevamos para ver más lejos a pesar de nuestra propia pequeñez".

Carpeta 3. Proveniencia: Londres, Bibl. brit., mss. Add. 24189, f. 15ro ; Harley 3647, f. 57ro ${ }^{\circ}$ 58vo. Descriptor: "Contra el tópico que hace de la Edad Media una época ignorante y supersticiosa, los especialistas conocen numerosos ejemplos de sus ideas científicas. Aunque definidas y organizadas de modo diferente al nuestro, las ciencias del Medievo no sólo requirieron la conservación y transmisión de la Filosofía antigua o el saber de otras culturas, sino que supusieron el desarrollo de nuevos conceptos e instrumentos como medio para afrontar retos técnicos y problemas puramente especulativos".

Carpeta 4. Proveniencia: Londres, Bibl. brit., mss. Harley 3667, f. 8ro y vo; Arundel 83, f. 123 vo. Descriptor: "Los cosmógrafos del Medievo se empeñaron en la descripción del Universo como parte de un esfuerzo más general por comprender la inserción del hombre en su entorno. Más allá del mito de la 'Tierra plana' que claramente desmienten, tales representaciones exceden lo puramente geográfico y están imbuidas por un profundo énfasis simbólico y alegórico. Son, asimismo, una buena clave para entender la Historia, la Filosofía, la Teología o las obras plásticas de la época”.

Carpeta 5. Proveniencia: Londres, Bibl. brit., mss. Royal 6E IX f. 29ro; Sloane 1712, f. 36ro; Oxford, Coll. S. Ioan. ms. 17 f. 7ro. Descriptor: "La clasificación y representación de las distintas formas de conocimiento constituye uno de los temas más interesantes 
de investigación para los medievalistas. Aunque se modificó ligeramente con el tiempo a partir de algunas reflexiones metodológicas, el esquema básico permaneció prácticamente inalterado por más de mil años, determinando el ordo lectionis o el curriculum en las escuelas y universidades. Desde tales supuestos, la Edad Media incluso desarrolló asombrosas técnicas pedagógicas encaminadas a la absorción y transmisión de dichos saberes".

Carpeta 6. Proveniencia: Lisboa, Bib. nat. Alcobacensis 426 f. $251 \mathrm{v}^{\text {o}}$; Londres, Bib. brit. mss. Harley 273, f. 112ro; Egerton 3314, f. $73 \mathrm{v}^{\circ}$. Descriptor: "El empleo de instrumentos que automatizan las tareas cotidianas no es prerrogativa de nuestra sociedad industrial. Como el de otras épocas, el hombre medieval desarrolló numerosas máquinas e ingenios destinados a facilitar su vida y trabajo, aunque también supo aprovechar su propio cuerpo en un modo sorprendente para resolver diversas tareas prácticas e intelectuales, justo como solemos hacer nosotros con nuestros computadores y dispositivos digitales".

\section{Referencias}

Barrows, H. (1988). The tutorial process. Southern Illinois University School of Medicine.

Bender, W. (2012). Project-based learning. Differentiating instruction for the 21st century. Corwin.

Carlino, P. (2003). Leer textos científicos y académicos en la educación superior: obstáculos y bienvenidas a una cultura nueva (1). Uni-pluri/versidad, 3(2), 17-23.

Castello, J. A. (2016). Historia de la Filosofía Medieval. [Programa de curso]. Facultad de Filosofía y Letras, Universidad de Buenos Aires. http:// repositorio.filo.uba.ar:8080/bitstream/handle/ filodigital/3853/uba_ffyl_p_2016_fil_Historia\%20 de\%20la\%20Filosof\%C3\%ADa\%20Medieval\%20 \%28Castello\%29.pdf

Covarrubias, A. (2012) Historia de la Filosofía Medieval. [Programa de curso]. Facultad de Filosofía-Instituto de Filosofía, Pontificia Universidad Católica de Chile http://filosofia.uc.cl/images/FIL123T_Sec.1_ Covarrubias.pdf

D’Amico, C. (2015). La Filosofía Medieval en Iberoamérica en el siglo xx. En M. R. Mate, O. Guariglia y L.
Olivé (ed.), Filosofía iberoamericana del siglo xx (I). Filosofía teórica e Historia de la Filosofía (pp. 433468). Trotta-Csic.

Gallagher, S. (1997). Problem-Based Learning: Where Did It Come From, What Does It Do, and Where Is It Going? Journal for the Education of the Gifted, 20(4), 332-362.

Gallagher, S. (2009). Problem-based learning. En J. S. Renzulli et al. (eds.), Systems and models for developing programs for the gifted and talented (pp. 193-210). Creative Learning Press.

Goh, K. (2012). A staff education and development programme to support PBL. En G. O'Grady, H. Yew, K. Goh y H. Schmidt (eds.), One-day, one-problem. An approach to problem-based learning (pp. 259-518). Springer.

Green, M. H., Walker-Meikle, K. y Müller, W. P. (2014). Diagnosis of a "Plague" Image: A Digital Cautionary Tale. The Medieval Globe, 1(1), 310-326.

Gutiérrez-Girardot, R. (2002). La filosofía en Hispanoamérica. Cuadernos Hispanoamericanos, 630, 81-88.

Leary, H., Walker, A., Shelton, B. y Fitt, M. (2015). Exploring the relationships between tutor background, tutor training, and student learning: a problem-based learning meta-analysis. En A. Walker, H. Leary, C. Hmelo-Silver y P. Ertmer (eds.), Essential readings in problem-based learning. Exploring and extending the legacy of Howard S. Barrows (pp. 381406). Purdue University.

Li, M. (2012). Approaches to learning: literature review. International Baccalaureate Organization.

McCaughan, K. (2015). Theoretical anchors for Barrows' PBL tutor guidelines. En A. Walker, H. Leary, C. Hmelo-Silver y P. Ertmer (eds.), Essential readings in problem-based learning. Exploring and extending the legacy of Howard S. Barrows (pp. 74-88). Purdue University.

Marenbon, J. (2007). Latin Averroism. En A. Akasoy, J. E. Montgomery y P. E. Pormann (eds.), Islamic crosspollinations: interactions in the Medieval Middle East (pp. 135-147). E. J. W. Gibb Memorial Trust.

Peen, T. y Arshad, M. (2014) Teacher and student questions: a case study in Malaysian secondary school problem-based learning. Asian Social Science, 10(4), 174-182.

Peterson, M. (1997). Skills to enhance problem-based learning. Medical Education Online, 2(1), 4289. 
Pierantoni, C. y Chávez, P. (2014). Seminario de Filosofía Medieval. [Programa de curso]. Facultad de Filosofía y Humanidades, Universidad de Chile. https://ucampus.uchile.cl/m/filosofia_catalogo/ programa?bajar $=1 \& \mathrm{id}=5627$

Priani, E. (2018). Historia de la filosofía 3: Edad Media. [Programa de curso]. Facultad de Filosofía y Letras, Universidad Nacional Autónoma de México. Recuperado de https://www.dropbox.com/s/ 16am2xnlaz2dfv1/HISTORIA-3_ERNESTOPRIANI. $\mathrm{pdf} ? \mathrm{dl}=0$

Redondo, J. (2018). Historia de la Filosofía III (Edad Media). Razón. Revelación y fe. Las vías del conocimiento en las filosofías medievales cristiana, islámica y judía. [Programa de curso]. Facultad de Filosofía y Letras, Universidad Nacional Autónoma de México. https://www.dropbox.com/s/4c5souj8mqkp6xa/HISTORIA\%20DE\%20LA\%20 FILOSOF\%C3\%8DA\%203_JOSE\%20MANUEL\%20 REDONDO\%20ORNALAS.pdf?

Rossi, A., Zea, L., Balcárcel, J.L. y Villegas, A. (1968). El sentido actual de la filosofía en México. Revista de la Universidad de México, XXII(5), IV-v.
Saranyana, J. (2004). La Filosofía Medieval en Latinoamérica. Universitas Philosophica, 21(42), 75-86.

Universidad del Cauca. (2013). Plan de Estudios. Filosofía. Facultad de Ciencias Humanas y Sociales. Popayán. https://www.unicauca.edu.co/fchs/ filosofia/plan-estudios

Universidad del Valle [Oficina de Planeación y Desarrollo Institucional]. (2017). Encuesta socioeconómica 2016. Autor

White, H. (2001). A PBL course that uses research articles as problems. En B. Duch, S. Groh y D. Allen (eds.), The power of problem-based learning (pp. 131-140). Sterling: Stylus.

Williams, J. (2012). Teachers as facilitators. En G. O'Grady, H. Yew, K. Goh y H. Schmidt (eds.), Oneday, one-problem. An approach to problem-based learning (pp. 237-258). Springer.

Zumthor, P. (1980). Parler du Moyen Âge. Les Éditions du Minuit. 